\title{
The AP-1 Transcription Factor Is a Key Determinant of Human Cytomegalovirus Latency and Reactivation ${ }^{\dagger}$
}

\author{
Benjamin A. Krishna, Amanda B. Wass and Christine M. O'Connor * \\ Lerner Research Institute, Cleveland Clinic, Cleveland, OH 44195, USA; krishnb2@ccf.org (B.A.K.); \\ wassa@ccf.org (A.B.W.) \\ * Correspondence: oconnoc6@ccf.org \\ † Presented at Viruses 2020-Novel Concepts in Virology, Barcelona, Spain, 5-7 February 2020. \\ Published: 13 July 2020
}

\begin{abstract}
Human cytomegalovirus (HCMV) is a ubiquitous pathogen that latently infects hematopoietic progenitor cells (HPCs). Individuals with a competent immune system are, for the most part, asymptomatic for the disease. However when a latently infected individual becomes immunosuppressed, HCMV can reactivate, causing severe morbidity and mortality. While much of the viral genome is transcriptionally silenced during latency, some genes are expressed, including the HCMV-encoded G-protein coupled receptor US28. We showed that US28 expression is required for latency, as it suppressed the activator protein-1 (AP-1) transcription factor by attenuating the AP-1 subunit, fos. In turn, this prevents AP-1 from binding and activating the major immediate early promoter (MIEP), the key promoter regulating the latent-to-lytic transcriptional "switch". Our new data suggest that US28-mediated signaling during latency attenuates the Src-MAPK signaling axis to regulate AP-1. We find that US28 expression suppresses Src, MEK, and ERK, as well as fos phosphorylation and AP-1 binding to the MIEP. Conversely, the pharmacological inhibition of Src, MEK, or ERK in US28A-latently infected HPCs suppresses infectious virus production, demonstrating the important role for this signaling axis during latency. Our recent data also reveal that regulating AP-1 is a key determinant in balancing HCMV latency and reactivation. Infection with a virus in which we disrupted the proximal AP-1 binding site in the MIEP (AP-1 $\Delta p$ ) leads to reduced AP-1 binding and inefficient viral reactivation compared to wild type. Furthermore, AP-1 is critical for the de-repression of MIEP-driven transcripts and downstream early and late genes, while other immediate early genes remain unaffected. Collectively, these data suggest that AP-1 binding to the MIEP is suppressed during latency, but is required for the efficient transactivation of the MIEP during reactivation. We are currently elucidating US28's involvement in recruiting AP-1 to the MIEP during reactivation.
\end{abstract}

Keywords: HCMV; cytomegalovirus; latency; reactivation; AP-1; US28; MAPK; MIEP

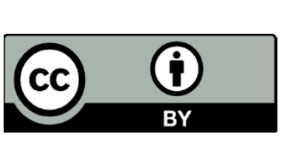

(C) 2020 by the authors. Licensee MDPI, Basel, Switzerland. This article is an open access article distributed under the terms and conditions of the Creative Commons Attribution (CC BY) license (http://creativecommons.org/licenses/by/4.0/). 